\section{Transfusión sanguínea y Testigos de Jehová: puntos a considerar}

\section{Blood transfusions and Jehová's Witnesses}

\section{Sr. Editor:}

Hemos leído con mucho interés el artículo publicado por el Sr. Retamales y el Dr. Cardemil referente a transfusiones sanguíneas y consentimiento informado, tomando como ejemplo a los pacientes Testigos de Jehová ${ }^{1}$. Estamos de acuerdo con los autores en la necesidad de que nuestros cuidados médicos sean tan seguros como respetuosos de la voluntad y dignidad de los pacientes. En este mismo sentido nos parece necesario realizar un par de comentarios con la intención de aportar a la discusión de este interesante tema:

$1^{\circ}$ Durante los últimos años ha aumentado la información sobre las complicaciones derivadas de la transfusión de productos sanguíneos ${ }^{2,3}$. Debido a esto, las técnicas de ahorro de sangre son cada vez más difundidas y actualmente utilizadas de manera habitual; tanto se ha avanzado en la materia que, en algunos casos, es posible llevar a cabo cirugías sin sangre que antaño parecían requerirla obligadamente. Sin embargo, también se ha acumulado evidencia sobre las consecuencias negativas que produce la anemia severa ${ }^{4,5} \mathrm{y}$, pese a los promisorios avances de la ciencia, aún no se encuentra disponible para uso clínico ningún sustituto sanguíneo artificial capaz de transportar oxígeno ${ }^{6}$ o promover la coagulación. Así, la administración de productos sanguíneos sigue siendo una de las alternativas terapéuticas de la medicina de hoy. Como todo tratamiento, tiene indicaciones, cada vez más acotadas y definidas, y efectos secundarios, cada vez más conocidos.

$2^{\circ}$ La autonomía es uno de los principios básicos de la bioética moderna. Existe consenso en que el respeto a la dignidad de las personas incluye acoger la voluntad de las mismas. En medicina esto se expresa por medio del consentimiento informado. Si bien no se pueden limitar los esfuerzos por defender este principio, no se puede llegar al punto de transferir la responsabilidad de las decisiones que son propias del médico al paciente excusándose diciendo "era lo que él quería". La responsabilidad moral por los actos es personal e intransferible. Por lo tanto, el médico debe coincidir con el paciente en cuál es la mejor alternativa terapéutica en cada caso en particular; de no existir acuerdo, el paciente puede abstenerse de recibir el tratamiento propuesto y/o el médico puede plantear las objeciones de conciencia que se le generen ${ }^{7}$.

Este tipo de casos nos recuerdan dos dimensiones esenciales de la práctica médica: la técnica, para avanzar en el conocimiento de las indicaciones y complicaciones de las transfusiones (así como de eventuales sustitutos efectivos) y la ética, para avanzar en el respeto necesario para relacionarnos con personas que pueden pensar diferente a nosotros.

Drs. Rodrigo López y Guillermo Lema Departamento de Anestesiología, Facultad de Medicina, Pontificia Universidad Católica de Chile. Santiago, Chile.

\section{Referencias}

1.- Retamales A, Cardemil G. Beneficios del ejercicio de la autonomía y consentimiento informado: Ejemplo de los Testigos de Jehova. Rev Med Chile 2009; 137: 1388-94.

2.- Hébert P, Wells G, Blajchman M, Marshall J, Martin C, Pagliarello G, et al. A Multicenter, randomized, controlled clinical trial of transfusion requirements in critical care. N Engl J Med 1999; 340 (6): 409-17.

3.- Murkin J. Transfusion Trigger Hct 25\%: Above or Below, Which is Better? Pro: Hct $<25 \%$ is better. J Cardiothor Vasc Anesth 2004; 18 (2): 234-37.

4.- Wu W, Rathore S, Wang Y, Radford M, Krumholz H. Blood transfusions in elderly patients with acute myocardial infraction. N Engl J Med 2001; 345 (17): 1230-36.

5.- Wall M. Transfusion Trigger Hct 25\%: Above or Below, Which is Better? Con: Hct $>25 \%$ is better. J Cardiothor Vasc Anesth 2004; 18 (2): 238-41.

6.- Weiskopf, Richard. Hemoglobin-Based Oxygen Carriers: Compassionate Use and Compassionate Clinical Trials. Anesth Analg 2010; 110 (3): 659.

7.- Casas L, Dides C. Objeción de conciencia y salud reproductiva en Chile: dos casos paradigmáticos. Acta Bioethica 2007; 13 (2): 199-206.

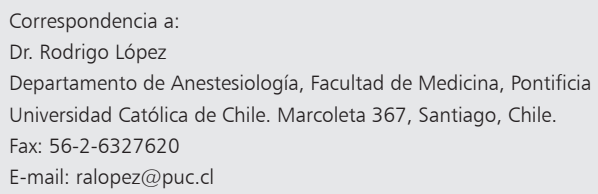




\section{$\underline{\text { Réplica de los autores aludidos }}$}

\section{Authors' reply}

\section{Sr. Editor:}

Resulta satisfactorio que exista reconocimiento general en orden a que los pacientes pueden optar a someterse a cirugías sin sangre, así como que ésa es una opción clínica (que se encuentra validada precisamente porque la transfusión de productos sanguíneos conlleva una serie de riesgos, muchos de ellos ya descritos y debidamente documentados).

La referencia que hacen los autores corresponsales a que, en todo caso, no puede descartarse la administración de productos sanguíneos como una alternativa terapéutica, confirma que ésta también es una opción clínica. Ello reafirma lo señalado en el punto precedente.

Finalmente, y no obstante estimar que el concepto y la idea de consentimiento informado debe tratarse en más líneas de las que permite un intercambio de correspondencia como el que nos reúne, es dable anotar que en una cosa estamos todos de acuerdo: "Siempre y en todo caso deben respetarse la dignidad y derechos de los pacientes".

Sr. Avelino Retamales P. ${ }^{1}$, Dr. Gonzalo Cardemil H. ${ }^{2}$ ${ }^{1}$ Departamento de Información sobre Hospitales-Chile para Testigos de Jehová. ${ }^{2}$ Unidad Cirugía de Tórax, Hospital Clínico de la Universidad de Chile. Santiago de Chile. 